\title{
Influence of different factors on the crystallization of TI 2223/LAO superconducting films deposited by spray pyrolysis
}

\author{
H. Nguyen Xuan ${ }^{1}$, S. Beauquis ${ }^{1}$, Ph. Galez ${ }^{1}$, D. De-Barros ${ }^{2}$, F. Weiss ${ }^{2}$, \\ M. Decroux ${ }^{3}$ and M. Therasse ${ }^{3}$
}

${ }^{1}$ LAIMAN - ESIA, Université de Savoie, BP. 806, 74016 Annecy Cedex, France

${ }^{2}$ LMGP, INPG, BP. 46, 38402 Saint-Martin-d'Hères Cedex, France

${ }^{3}$ DPMC, Université de Genève, 24 Quai Ernest-Ansermet, 1211 Genève 4, Suisse

\begin{abstract}
The elaboration of Tl-2223 films by a two step process has been investigated. The films contain both Tl-2212 and Tl-2223 phases. We find that the kinetics for the Tl-2212 O Tl-2223 transformation is very slow due to the texture of the Tl-2212 phase. Critical current densities up to 1.3 $\mathrm{MA} / \mathrm{cm}^{2}$ (self-field, $77 \mathrm{~K}$ ) are however measured. Pure Tl-2223 is obtained when the films are thallinated with a fluorinated source of thallium.
\end{abstract}

\section{INTRODUCTION}

High $T_{c}$ Superconductors (HTS) have many advantages for applications in microwave devices. One of these applications concerns passive filters for wirelesses telecommunications. It is based on superconducting films $\left(\mathrm{YBa}_{2} \mathrm{Cu}_{3} \mathrm{O}_{7}\left(T_{c}=92 \mathrm{~K}\right)\right.$ or $\left.\mathrm{Tl}_{2} \mathrm{Ba}_{2} \mathrm{CaCu}_{2} \mathrm{O}_{8}\left(T_{c}=105 \mathrm{~K}\right)\right)$ deposited on a single crystal substrate $\left(\mathrm{LaAlO}_{3}, \mathrm{SrTiO}_{3}, \mathrm{MgO}, \mathrm{Al}_{2} \mathrm{O}_{3} / \mathrm{CeO}_{2}, \ldots\right)$. These devices are already used in the realization of base stations. In the family of Tl-based cuprates, the $\mathrm{Tl}_{2} \mathrm{Ba}_{2} \mathrm{Ca}_{2} \mathrm{Cu}_{3} \mathrm{O}_{10}$ phase (Tl-2223) is interesting because of its high critical temperature, $T_{c}=123 \mathrm{~K}$ measured on bulk samples prepared by solid state reaction in a closed system. Its use should result in a significant lowering of the cost of cryogenics [1-8]. The development of superconducting Tl-2223 films is currently realized by a two steps process: (1) the deposition of a precursor film with composition $\mathrm{Ba}: \mathrm{Ca}: \mathrm{Cu}=2: 2: 3$ by spray pyrolysis and (2) the ex-situ thallination by a solid-gas reaction in a closed system. Previous studies by Phok et al. [3] and Holstein et al. [5] have shown the possibility of preparing Tl-2223/LAO films with good superconducting properties $T_{c} \approx 120 \mathrm{~K}, J_{c}=0.7 \mathrm{MA} / \mathrm{cm}^{2}$. The films have indeed a very low surface resistance, 6.5 P: [at $77 \mathrm{~K}$ and $87 \mathrm{GHz}$ [9]. However the preparation of pure Tl-2223 films with smooth surfaces is not yet controlled. We report here some results on Tl-2223 film preparation. We discuss about the influence of the texture of films on the reaction time and especially on the Tl22120 Tl-2223 transformation. The influence of fluorine is also presented. 


\section{EXPERIMENTAL}

Precursor films with composition $\mathrm{Ba}_{2} \mathrm{Ca}_{2} \mathrm{Cu}_{3} \mathrm{O}_{z}$ are deposited by spray pyrolysis on a $\mathrm{LaAlO}_{3}$ (LAO) substrate (100) oriented $\left(5 \times 5 \times 0.5 \mathrm{~mm}^{3}\right)$, starting from a solution of barium, calcium and copper nitrates with overall concentration $0.3 \mathrm{~mol} / \mathrm{L}$ according to the procedure described by Phok et al. [3, 10]. These precursor films are then thallinated in a closed system with a $\mathrm{Tl}_{x} \mathrm{Ba}_{2} \mathrm{Ca}_{2} \mathrm{Cu}_{3} \mathrm{O}_{z}(x \approx 2)$ pellet as a source of thallium. The treatments with annealing time ranging from 30 minutes to 30 hours were carried out between $1140 \mathrm{~K}$ and $1170 \mathrm{~K}$. The $\mathrm{Tl}_{x} \mathrm{Ba}_{2} \mathrm{Ca}_{2} \mathrm{Cu}_{3} \mathrm{O}_{z}\left(\mathrm{~F}_{x}\right)$ source was obtained by solid state reaction starting from the oxides and TlF. $\mathrm{BaO}_{2}, \mathrm{CuO}$ and $\mathrm{CaO}$ are first calcinated at $1223 \mathrm{~K}$ for 12 hours Then, after $\mathrm{Tl}_{2} \mathrm{O}_{3}$ or $\mathrm{TlF}$ addition in the appropriate proportion, the powders are pressed into pellets and heated at $1073 \mathrm{~K}$ for 6 hours under flowing oxygen. Bulk samples with $\mathrm{Tl}_{x} \mathrm{Ba}_{2} \mathrm{Ca}_{2} \mathrm{Cu}_{3} \mathrm{O}_{z}$ composition $(x \approx 2)$ were also prepared in a closed system at $1148 \mathrm{~K}$ by solid state reaction.

The surface morphology and the composition of the precursor and the thallinated films were characterized by scanning electron microscopy (SEM) coupled with energy dispersive $\mathrm{X}$-Ray spectroscopy (EDX). The identification and the quantification of the phases present in the films were carried by X-ray diffraction in the Bragg-Brentano geometry on a Philips PW1150 goniometer, $(\mathrm{Cu} \mathrm{KD})$. The orientation of the superconducting phases in $a-b$ plane and the $c$-axis orientation were determined by $M_{\text {scan }}$ and $Z$-scan measurements with a texture goniometer (Siemens D500 ( $\mathrm{Cu} \mathrm{KD)}$ ). Finally, $A C$ susceptibility and third harmonic $\left(V_{3}\right)$ measurements $[11,12]$ were used to determine the critical temperature $T_{c}$ and the critical current densities $J_{c}$ of the superconducting phases.

\section{RESULTS AND DISCUSSION}

\section{1 $\quad \mathrm{Ba}_{2} \mathrm{Ca}_{2} \mathrm{Cu}_{3} \mathrm{O}_{z}$ precursor films deposited by spray pyrolysis}

The morphology of the $\mathrm{Ba}_{2} \mathrm{Ca}_{2} \mathrm{Cu}_{3} \mathrm{O}_{z}$ precursor films after deposition at $1123 \mathrm{~K}$ for 18 minutes is homogeneous with submicronic grains of $\mathrm{BaCuO}_{2}, \mathrm{CaO}$ and $\mathrm{Ba}_{2} \mathrm{Cu}_{3} \mathrm{O}_{5+G}$. On the X-ray diffraction pattern (not shown here), we can observe that the $\mathrm{BaCuO}_{2}$ phase is already textured on the (100) plane of the LAO substrate. The presence of the three compounds, $\mathrm{BaCuO}_{2}, \mathrm{Ba}_{2} \mathrm{Cu}_{3} \mathrm{O}_{5+G}$ and $\mathrm{CaO}$ in he films indicates that the thermodynamic equilibrium is not yet reached. A subsequent annealing under flowing oxygen at $1123 \mathrm{~K}$ for 12 hours leads to the thermodynamic equilibrium with $\mathrm{BaCuO}_{2}, \mathrm{Ca}_{2} \mathrm{CuO}_{3}$ and $\mathrm{CaO}$ [13]. It can be deduced that he composition of the precursor films is very close to the expected one. This point is of course crucial for the purity of the final superconducting layers.

\subsection{Tl-2223 films prepared with a fluorine free thallium source $\mathrm{Tl}_{\mathrm{x}} \mathrm{Ba}_{2} \mathrm{Ca}_{2} \mathrm{Cu}_{3} \mathrm{O}_{z}$}

Preliminary studies showed that, in a closed system, the pathway for the formation of the Tl-1223 and Tl-2223 phases in a bulk samples or in films $[2-4,14]$ is the following: precursor/oxides $0 \quad \mathrm{Tl}_{2} \mathrm{BaO}_{4}$ o $\mathrm{Tl}_{6} \mathrm{Ba}_{4} \mathrm{O}_{13}$ O $\mathrm{Tl}-2212$ O Tl-2223 O Tl-1223.

The Table 1 presents the compositions of bulk samples and thallinated films in function of annealing time. Both kind of samples were reacted at $1148 \mathrm{~K}$ under the same conditions. The proportion of superconducting phases were evaluated by X-ray diffraction by comparing of the intensities of the three most intense peaks of each phase. 
Table 1. Estimated proportion of superconducting phases in bulk samples and thallinated films reacted at $1148 \mathrm{~K}$ as a function of annealing time.

\begin{tabular}{|c|c|c|c|c|c|c|}
\hline \multirow{2}{*}{$\begin{array}{c}\text { Time } \\
\text { (min.) }\end{array}$} & \multicolumn{3}{|c|}{ Film samples } & \multicolumn{3}{c|}{ Bulk samples } \\
\cline { 2 - 7 } & Superconducting phases (\%) & \multicolumn{2}{c|}{ Superconducting phases (\%) } \\
\hline 30 & 0 & 100 & 0 & 0 & 100 & 0 \\
\hline 60 & 0 & 100 & 0 & 5 & 95 & 0 \\
\hline 180 & 35 & 65 & 0 & 33 & 67 & 0 \\
\hline 360 & 50 & 45 & 5 & 77 & 23 & 0 \\
\hline 720 & 20 & 60 & 20 & 97 & 3 & 0 \\
\hline 1080 & - & - & - & 75 & 0 & 25 \\
\hline 1440 & - & - & - & 50 & 0 & 50 \\
\hline 1800 & 30 & 35 & 35 & - & - & - \\
\hline
\end{tabular}

In the case of films, the maximum Tl-2212 o Tl-2223 transformation (approximately 50\% Tl-2223) is obtained for a 6 hour treatment. Then the transformation Tl-2223 o Tl-1223 begins presumably because of a decrease in $\mathrm{Tl}_{2} \mathrm{O}$ vapor due to some reaction with quartz. The results are significantly different for bulk samples. Indeed, the Tl-2223 phase with a purity up to $97 \%$ is obtained at 720 minutes $\left(T_{c}=123 \mathrm{~K}\right)$. After 720 minutes, the Tl-2223 o Tl-1223 decomposition starts, and no more Tl-2212 phase can be detected.

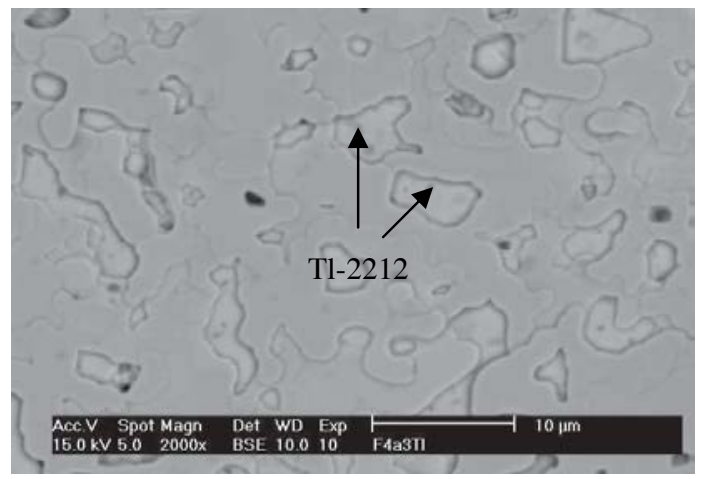

Figure 1. BSE image of a film thallinated with a pellet $\mathrm{Tl}_{x} \mathrm{Ba}_{2} \mathrm{Ca}_{2} \mathrm{Cu}_{3} \mathrm{O}_{z}(x \approx 2)$ at $1148 \mathrm{~K} / 180 \mathrm{~min}$.

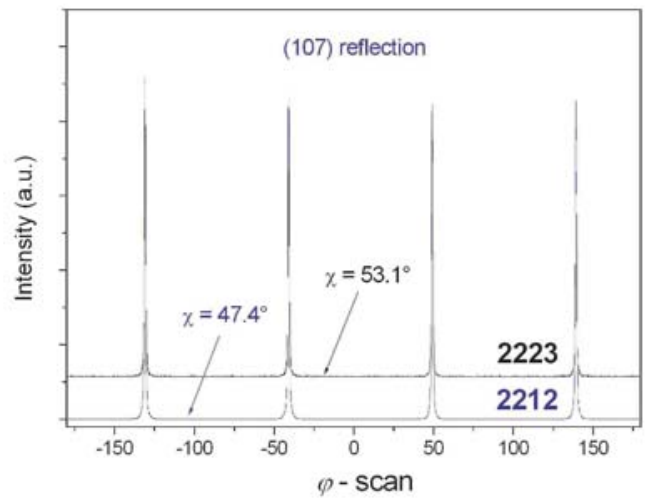

Figure 2. Phi-scan of the 107 reflection of the Tl2223 and Tl-2212, for a film thallinated at $1148 \mathrm{~K} /$ $180 \mathrm{~min}$.

Figure 1 presents the backscattered electron (BSE) image of a film thallinated during 180 minutes. Grains of Tl-2223 phase with size larger than $5 \mathrm{Pm}$ cover the surface. The Tl-2212 phase (brighter) seems to be located below the Tl-2223 phase. This is consistent with X-ray diffraction results which show larger Tl-2223 peaks at low angles and larger Tl-2212 peaks at intermediate angles.

The biaxial texture of both Tl-2212 and Tl-2223 phases is demonstrated by phi-scan (107 reflection) and $Z$-scan (00,12 and 00,14 reflection, respectively) measurements. Figure 2 presents the phi-scan 
measurements on a film thallinated during 180 minutes. The full width at half-maximum are: $\mathrm{FWHM}_{(\mathrm{Tl}-2212)}=0.49^{\circ}$ and $\mathrm{FWHM}_{(\mathrm{Tl}-2223)}=0.46^{\circ}$. Characterizations by Z-scan on this film gives $\mathrm{FWHM}_{(\mathrm{Tl}-2212)}=0.37^{\circ}$ and $\mathrm{FWHM}_{(\mathrm{Tl}-2223)}=0.44^{\circ}$. These results highlight epitaxial growth of the two phases on the film.

The critical temperature measurement by $A C$ susceptibility of such a film shows clearly two transitions. One corresponds to the Tl-2212 phase $\left(T_{c}=106 \mathrm{~K}\right)$, the other is characteristic of Tl-2223 phase $\left(T_{c}=116 \mathrm{~K}\right)\left(\right.$ Fig. 3). This film has a $J_{c}$ of $1.2 \mathrm{MA} / \mathrm{cm}^{2}$ at $77 \mathrm{~K}$, in self field (Fig. 4).

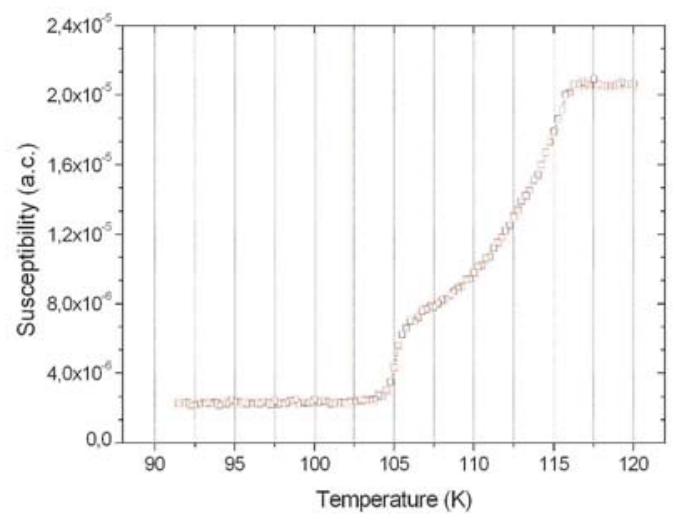

Figure 3. $T_{c}$ measured by $A C$ susceptibility of a film thallinated at $1148 \mathrm{~K} / 360 \mathrm{~min}$.

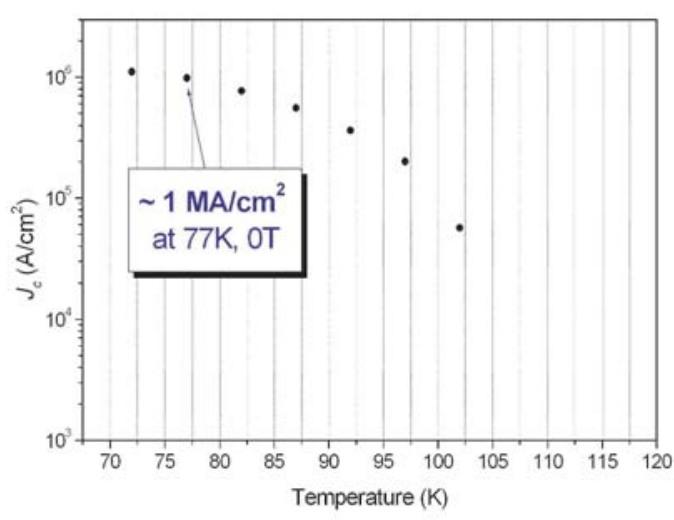

Figure 4. Critical current density $J_{c}$ as a function of temperature.

Until now we did not succeed to obtain the pure Tl-2223 films. The problem can have several origins. One possibility is that the composition of precursor film is probably not rich enough in calcium and copper, favoring the Tl-2212 formation at thermodynamic equilibrium. But, this is probably not sufficient to explain the difficulties in forming the Tl-2223 phase. It seems that the kinetics of the Tl-22120 Tl-2223 transformation is particularly slow for films. We think that the Tl-2212 o Tl-2223 transformation implies a two dimension diffusion mechanism of calcium and copper in the basal plane of the Tl-2212 structure. If the Tl-2212 phase is highly textured with large grains, then there are very few locations where $\mathrm{Ca}, \mathrm{Cu}$ and $\mathrm{O}$ atoms can step into the structure of Tl-2212. Moreover, large distances have to be covered by these atoms. The transformation occurs very slowly from the outer surface (in contact with the pellet) so that it becomes even slower with increasing time. On the other hand, for very long reaction times, Tl-2223 decomposes into Tl-1223, because of a loss of thallium vapor, probably due to a reaction with quartz.

An convincing argument in favor of this hypothesis, is that the Tl-2223 phase with a purity up to $97 \%$ is obtained after a 720 minute annealing for bulk samples with no or little preferred orientation.

The only possibility to enhance the proportion of Tl-2223 phase is therefore to reduce the texture of the Tl-2212 intermediate phase during the formation path.

\subsection{Film Tl-2223 prepared with a fluorinated source of thallium $\mathrm{Tl}_{x} \mathrm{Ba}_{2} \mathrm{Ca}_{2} \mathrm{Cu}_{3} \mathrm{O}_{z} \mathrm{~F}_{x}$}

Pellets of $\mathrm{Tl}_{x} \mathrm{Ba}_{2} \mathrm{Ca}_{2} \mathrm{Cu}_{3} \mathrm{O}_{z} \mathrm{~F}_{x}$ as a source of thallium have already been used to obtain good quality Tl1223 film. The highest critical current densities were measured in these Tl-1223 films $\left(0.8 \mathrm{MA} / \mathrm{cm}^{2}\right)$ but unfortunately a reduction of the critical temperatures probably due to the presence of fluorine in the structure occurred $[3,10,15,16]$. 
Films thallinated with a source containing TIF (1148 K / $180 \mathrm{~min}$.) contain only the superconducting Tl-2223 phase and $\mathrm{BaF}_{2}$ as an impurity phase on the surface (Fig. 5-6). They are biaxially textured with ' $M=0.43^{\circ}$ and ' $Z=0.55^{\circ} . T_{c}$ is $105 \mathrm{~K}$ and the relatively low $J_{c}$ value $\left(\approx 1 \times 10^{5} \mathrm{~A} / \mathrm{cm}^{2}\right)$ is explained by the presence holes in the films. Indeed, fluorine involves a liquid phase which sticks together the films and the pellets. Nevertheless, we think that the fluorine addition decreases liquidus and solidus temperatures which favors Tl-2212 o Tl-2223 transformation. A slight decrease of the reaction temperature and/or the fluorine content should lower the quantity of liquid phase and finally prevent the formation of holes.

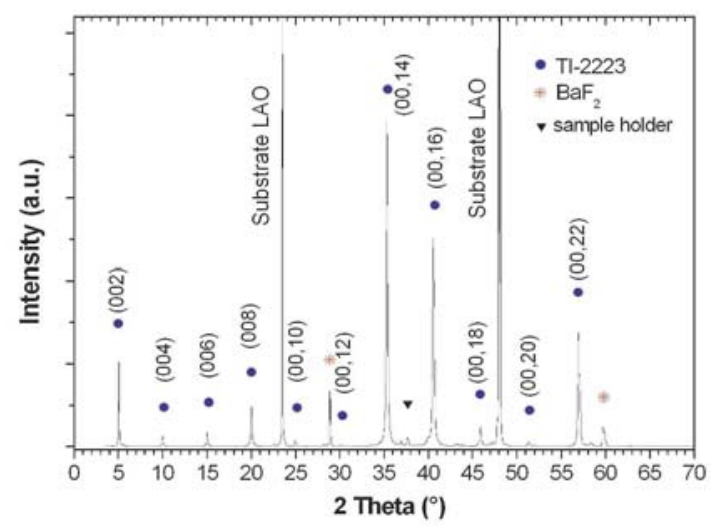

Figure 5. XRD $(T-2 T)$ of a Tl-2223/LAO film thallinated with a $\mathrm{Tl}_{x} \mathrm{Ba}_{2} \mathrm{Ca}_{2} \mathrm{Cu}_{3} \mathrm{O}_{z} \mathrm{~F}_{x}$ source $(x \approx 2)$.

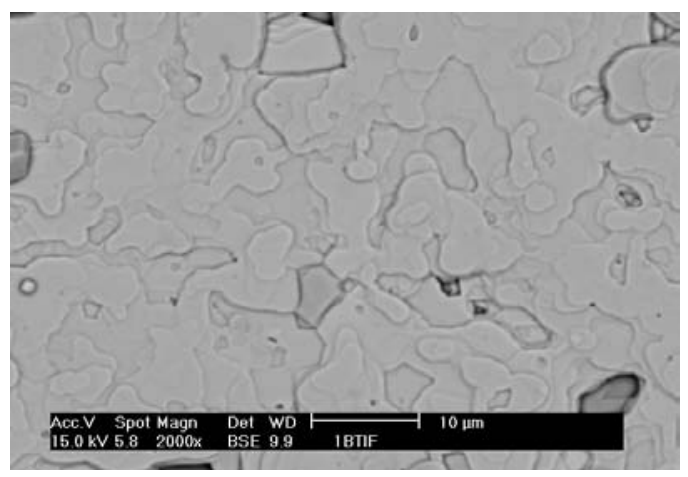

Figure 6. BSE image of a Tl-2223 film containing fluorine.

\section{CONCLUSIONS}

The elaboration of superconducting Tl-2223 / LAO films in a closed system leads to a mixture of two Tl-2223 and Tl-2212 phases with a maximum Tl-2223 fraction of 50\%. Our studies showed that the formation of Tl-2223 phase is limited by the annealing reaction time. If the annealing time is too long, an important loss of thallium in vapor phase occurs which leads to the decomposition of Tl-2223 into Tl-1223. Moreover, if the Tl-2212 phase is textured at the beginning of the Tl-2212 O Tl-2223 transformation, it becomes a barrier which prevent the formation of Tl-2223. This is related to the transformation mechanism which involves the two dimension diffusion of calcium and copper atoms in the basal plane of the Tl-2212 structure. However, these superconducting films have a $J_{c}$ up to 1.2 $\mathrm{MA} / \mathrm{cm}^{2}$ at $77 \mathrm{~K}$ in self field. These results are very promising for applications.

The effect of fluorine on the crystallization of the Tl-2223 films was also studied. It made it possible to obtain superconducting Tl-2223 films with a high purity. The presence of a liquid phase at low temperature during thallination favors the transformation from Tl-2212 to Tl-2223 but the appearance of holes results in a reduction of the superconducting qualities of the films.

\section{Acknowledgements}

This work supported by the Region Rhône-Alpes within the framework of the "Avenir" research program TOSCA. 


\section{References}

[1] G. Malandrino and I.L. Fragala, High Temperature Superconductivity 1 Materials pp. 169-211, Ed. A.V. Narlikar, Springer-Verlag: Berlin, Heidelberg, 2004

[2] J.L. Jorda, Ph. Galez, S. Phok, Th. Hopfinger, T.K. Jondo, High Temperature Superconductivity 1 Materials pp. 29-78, Ed. A.V. Narlikar, Springer-Verlag: Berlin, Heidelberg, 2004

[3] S. Phok, Z.I. Supardi, A. Sin, Ph. Galez, J.L. Jorda, F. Weiss, J. Phys. IV France 11 (2001) 157

[4] Ph. Galez, J.L. Soubeyroux, Th. Hopfinger, Ch. Opagiste, M. Lomello-Tafin, Ch. Bertrand, J.L. Jorda, Supercond. Sci. Technol. 14 (2001) 583

[5] W.L. Holstein, L.A. Parisi, Journal of Materials Research 11, $\mathbf{N}^{\circ} \mathbf{6}$ (1996) 1349

[6] W.L. Holstein, C. Winker, D.B. Laubacher, D.W. Face, P. Pang, M.S. Warrington, L.A. Parisi, Journal Appl. Phys. 74 (2) (1993) 1426

[7] Y. Xin, B.R. Xu, S. Nasrazadani, W.S. He, D.F. Lu, G.F. Sun, K.W. Wong, D. Knapp, Journal of Materials Research 9, N7 (1994) 1672

[8] G. Samadi Hosseinai, W. Straif, K. Kundzins, H.W. Weber, S.L. Yan, M. Manzel, E. Stang, S. Proyer, D. Bäuerle, E. Mezzetti, Physica C 268 (1996) 307

[9] S. Huber, M. Manzel, H. Bruchlos, S. Hensen, G. Müller, Physica C 244 (1995) 337

[10]S. Phok, Ph. Galez, J.L. Jorda, D. De-Barros, C. Villard, F. Weiss, Crystal Engineering V5, I3-5 (2002) 401

[11]Yasunori Mawatari, Hirofumi Yamasaki, and Yoshihiko Nakagawa, Applied Physics Letters, V81 $\mathbf{N}^{\circ} \mathbf{1 3}$ (2002) 2424

[12]J. H. Claassena, Applied Physics Letters,V84 N7 (2004) 1135

[13]H. Nguyen Xuan, C. Bertrand, S. Beauquis, A. Pisch and Ph. Galez (submitted to J. Phys. IV)

[14]Ph. Galez, Th. Hopfinger, O.O. Scherban, R.E. Gladyshevkii, M. Lomello-Tafin, J.L. Jorda, M. Couach, Journal of Alloys and Compounds 333 (2002) 237

[15]Manfred Enengl, Gerhard Gritzner, Supercond. Sci. Technol. 16 (2003) 956

[16]Kyoji Tachikawa, Akihiro Kikuchi, Kiyoshi Inoue, Physica C 337 (2000) 180 